\title{
Optical parametric oscillator based off-axis integrated cavity output spectroscopy for rapid chemical sensing
}

\author{
Denis D. Arslanov, * Simona M. Cristescu, and Frans J. M. Harren \\ Life Science Trace Gas Facility, Molecular and Laser Physics, Radboud University, \\ Heyendaalseweg 135, Nijmegen 6525AJ, The Netherlands \\ *Corresponding author: d.arslanov@science.ru.nl
}

Received August 2, 2010; accepted September 14, 2010;

posted September 16, 2010 (Doc. ID 132803); published September 30, 2010

\begin{abstract}
An optical parametric oscillator (OPO), pumped by a fiber-amplified diode laser, is combined with off-axis integrated cavity output spectroscopy (OA-ICOS). The cw OPO (power $1.2 \mathrm{~W}$, tunability $3-4 \mu \mathrm{m}, 5 \mathrm{~cm}^{-1}$ mode-hop-free tuning) has a tuning speed of $100 \mathrm{THz} / \mathrm{s}$, which is ideal for rapid and sensitive trace gas detection. Combined with OA-ICOS, a detection limit of 50 parts per trillion by volume $\left(1 \times 10^{12}\right)$ of ethane $\left(\mathrm{C}_{2} \mathrm{H}_{6}\right)$ in nitrogen was obtained in $0.25 \mathrm{~s}$ at $2997 \mathrm{~cm}^{-1}$, corresponding to a noise equivalent absorption sensitivity of $4.8 \times 10^{-11} \mathrm{~cm}^{-1} \mathrm{~Hz}^{-1 / 2}$. The system demonstrates real-time measurements of methane and water in exhaled human breath. (C) 2010 Optical Society of America

OCIS codes: $\quad 190.4970,190.4410,190.4360,300.6340,300.6390,300.6320$.
\end{abstract}

Trace gases are distinctive markers of biological processes occurring in plants, animals, and humans [1-3] An excellent method for highly sensitive and selective detection of these volatile organic compounds is laser spectroscopy in the mid-IR wavelength region; at that wavelength, many molecules have strong distinctive absorption patterns [2]. It is of most interest to capture gas-mixing concentrations at a subsecond time scale, e.g., to investigate the reaction of an insect or plant on an environmental change, but also to monitor during the single breath of a human subject. Next to blood, urine, or tissue sampling, the breath test can be important in yielding clinical information for diagnosis of diseased states or to monitor therapy [4]. The major advantage of analyzing on-line exhaled breath is that it is noninvasive, represents minimal risk, and can be sampled repetitively, thereby avoiding the risks taken during off-line gas analysis, in which systematic errors can be introduced during collection, concentration, and transport of the sample. Here, on-line analysis means that the sample is taken during the last part of the exhalation. Since this period is only a few seconds, we developed a fast-scanning $\mathrm{cw}$ OPO, pumped by a fiber-amplified diode laser $[\underline{[}, \underline{6}]$ in combination with a fast spectroscopic trace-gas detection scheme.

There are several basic laser-based spectroscopic techniques, such as photoacoustic spectroscopy (PAS), wavelength modulation spectroscopy, cavity ring-down spectroscopy (CRDS), and integrated cavity output spectroscopy (ICOS), also called cavity-enhanced absorption spectroscop. PAS can be very sensitive and efficient owing to its easy alignment and simple use; however, it cannot be used at a subsecond time scale [2,7]. Wavelength modulation [] is in general less sensitive than the cavity enhanced methods CRDS and ICOS. Within ICOS $[\underline{9}, \underline{10}]$, a gas-detection cell is used with two highly reflective end mirrors (>99.9\%), resulting in a very high finesse cavity [ratio between the free spectral range (FSR) and FWHM of the cavity mode: $F>8000$ ]. In contrast to cw CRDS [11], the laser light is not locked on each cavity mode but swept over the gas absorption line. Moreover, opposite to on-axis alignment at the center of the optical cav- ity, off-axis injection of the laser light (OA-ICOS) into the cavity will generate spatially separated, multiple reflections within the cavity before the reentrant condition of the optical beam is fulfilled. Therefore, OA-ICOS is effectively lowering the FSR of the cavity, producing a dense mode structure $[12,13]$. In a successful off-axis alignment, many cavity modes exist underneath every molecular transition and the laser linewidth should be broad with regard to the FSR of the cavity, but narrow with regard to the molecular line. Even narrowband lasers couple to many cavity modes simultaneously, which gives alignment robustness at the cost of cavity throughput power. One big advantage of OA-ICOS as compared with CRDS is that it can be used without limitations concerning ring-down time or mode matching between the laser frequency and the FSR.

Our experimental setup is shown on the Fig. 1 and is described elsewhere [5]. A $80 \mathrm{~mW}$ multisection distributed Bragg reflector $(\mathrm{D} \overline{\mathrm{B} R})$ diode laser (Eagleyard Photonics) with an output wavelength at $1082 \mathrm{~nm}$ is used to seed a 30-m-long double-clad Yb-doped fiber amplifier (F), which is pumped by a $25 \mathrm{~W}$ fiber-coupled $976 \mathrm{~nm}$ cw diode-laser bar (FP). Two isolators (ISO 1 and 2) are used to prevent any feedback, while a quarter-wave

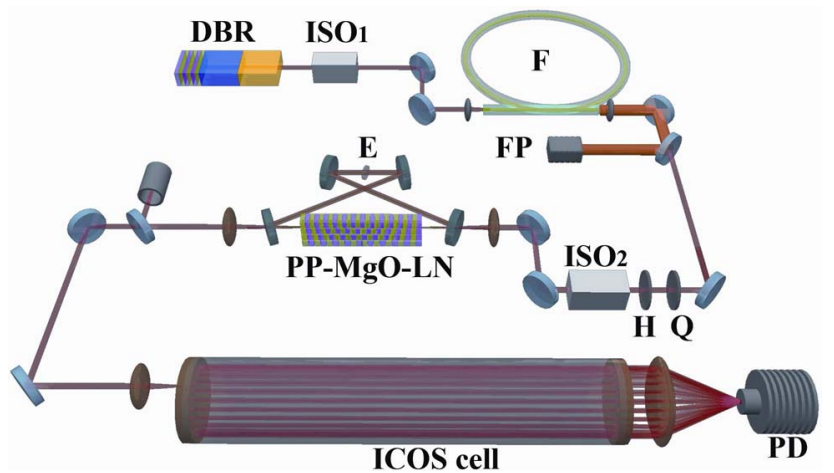

Fig. 1. (Color online) Experimental setup. DBR, distributed Bragg reflector diode laser; ISO 1 and 2, isolators; F, double-clad Yb-doped fiber; FP, fiber pump diode-laser bar; Q, quarter-wave plate; $\mathrm{H}$, half-wave plate; $\mathrm{PP}-\mathrm{MgO}-\mathrm{LN}, \mathrm{MgO}$-doped periodically poled lithium niobate crystal; E, intracavity etalon; ICOS cell, integrated cavity output spectroscopy cell; PD, photodetector. 
plate (Q) and half-wave plates $(\mathrm{H})$ are for polarization control. An output power of $7.5 \mathrm{~W}$ is then available. By synchronized tuning of the phase section of the DBR laser, $3 \mathrm{~cm}^{-1}$ wide mode-hop-free tuning ranges can be achieved at scanning rates of $1 \mathrm{kHz}$. Slower scanning rates will cover a wider wavelength range (e.g., $5 \mathrm{~cm}^{-1}$ at $100 \mathrm{~Hz}$ ). The effective output linewidth of the DBR laser is $100 \mathrm{MHz}$. The singly resonant cw OPO cavity consists of a four-mirror bow-tie ring design. The pump beam was focused in a $5 \%$ MgO-doped periodically poled lithium niobate crystal with seven poling periods ranging from 28.5 to $31.5 \mu \mathrm{m}$. The crystal was placed in an oven capable of maintaining temperatures from $20^{\circ} \mathrm{C}$ to $200{ }^{\circ} \mathrm{C}$ with a stability of $0.02{ }^{\circ} \mathrm{C}$. Up to $1.2 \mathrm{~W}$ of idler output was produced at a pump power of $7.5 \mathrm{~W}$.

For OA-ICOS, the high-finesse optical cavity was formed by a pair of highly reflective $(R \sim 99.98 \%$ at $3.3 \mu \mathrm{m}$, NovaWave Technology) 2 in. diameter $(1 \mathrm{in} .=2.54 \mathrm{~cm})$ concave $(R=1 \mathrm{~m})$ mirrors and a 60 -cm-long stainless steel tube with an inner diameter of $40 \mathrm{~mm}$ with two inlets/ outlets for gas exchange. The transmitted laser intensity was focused by an off-axis parabolic mirror (not shown) and a secondary lens $(f=5 \mathrm{~cm})$ to the fast (HgCdZnTe) thermoelectrically cooled photodetector (VIGO Systems PDI-2TE-4), which has a time response of $20 \mathrm{~ns}$.

The absorption is measured as an integrated signal from all the light transmitted through the cavity (see Fig. 1). We investigated if the signal-to-noise ratio (SNR) can be increased by aligning the cell more off-axis [12-14]. For that, each end of the cell was attached to an independent twodimensional translation stage. After proper on-axis alignment, both ends were translated orthogonally to the cavity axis in a horizontal direction collinear over the same length. As such, the laser beam draws a horizontal line on the mirror surfaces. Then the mirrors were translated vertically for the double length, but in opposite directions. In this case, the beam forms a circular pattern on each mirror with a diameter depending on the translation length. To study the offaxis alignment, we used a mixture of 20 parts per billion by volume (ppbv, $\left.1 \times 10^{9}\right)$ of ethane $\left(\mathrm{C}_{2} \mathrm{H}_{6}\right)$ in nitrogen, which
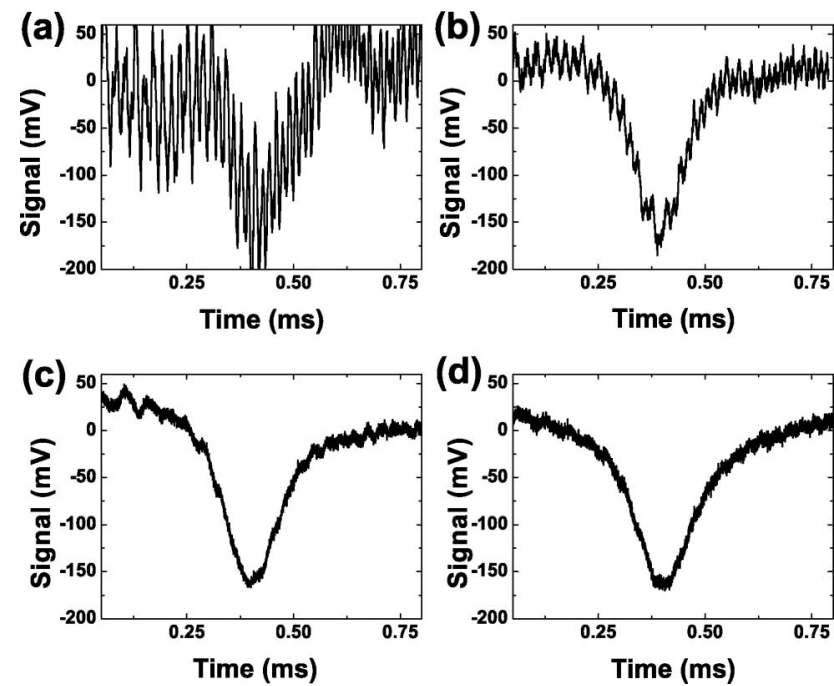

Fig. 2. 20 ppbv of ethane $\left(\mathrm{C}_{2} \mathrm{H}_{6}\right)$ mixture in nitrogen recorded in $0.25 \mathrm{~s}$ at a scanning rate of $1 \mathrm{kHz}$ and a wavelength of $2996.9 \mathrm{~cm}^{-1}$ for the cases (a) almost on-axis alignment, (b) $7 \mathrm{~mm}$ diameter off-axis alignment, (c) $21 \mathrm{~mm}$, and (d) $35 \mathrm{~mm}$. was continuously flushed through the cell with a flow speed of $5 \mathrm{l} / \mathrm{h}$, creating a homogenous distributed gas mixture at a constant pressure of $250 \mathrm{mbar}$. The pressure was kept at this constant value by using a pump, mass flow controllers, and a needle valve. The laser scans were made over an ethane absorption feature at $2996.9 \mathrm{~cm}^{-1}$. Various off-axis alignment distances were applied and the results are shown in Figs. 2 and 3(a). Each data point was recorded at a laser scanning rate of $1 \mathrm{kHz}$ and an integration time of $0.25 \mathrm{~s}$. Figure 2 shows clearly the difference between on-axis and off-axis alignment at 7, 21, and $35 \mathrm{~mm}$ diameter of the circular pattern. Figure 3(a) shows the increase of SNR, due to the fact that the signal stays at almost the same level for the different off-axis parameters, while the noise is decreased considerably.

The SNR can be also increased by increasing the scan speed of the laser. Figure 3(b) shows, at a fixed off-axis diameter $(35 \mathrm{~mm})$, the results of various scanning rates between $32 \mathrm{~Hz}$ and $2 \mathrm{kHz}$. The noise decreases when the signal is integrated over more scans in the fixed time frame of $0.25 \mathrm{~s}$. To determine the overall sensitivity of the OPO-based OA-ICOS setup, several ethane concentrations were recorded at $2996.9 \mathrm{~cm}^{-1}$. A linear response (not shown) allows determining a detection limit of 50 parts per trillion by volume (pptv, $1 \times 10^{12}$ ) of $\mathrm{C}_{2} \mathrm{H}_{6}$ measured in $0.25 \mathrm{~s}$. This corresponds to a noise equivalent absorption sensitivity [10] of $4.8 \times 10^{-11} \mathrm{~cm}^{-1} \mathrm{~Hz}^{-1 / 2}$, the lowest value achieved in the mid-IR region at around $3.3 \mu \mathrm{m}$ up to now.

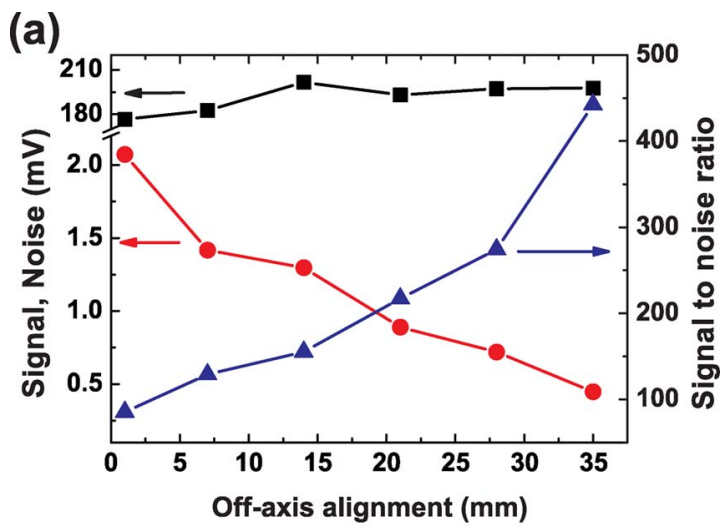

(b)

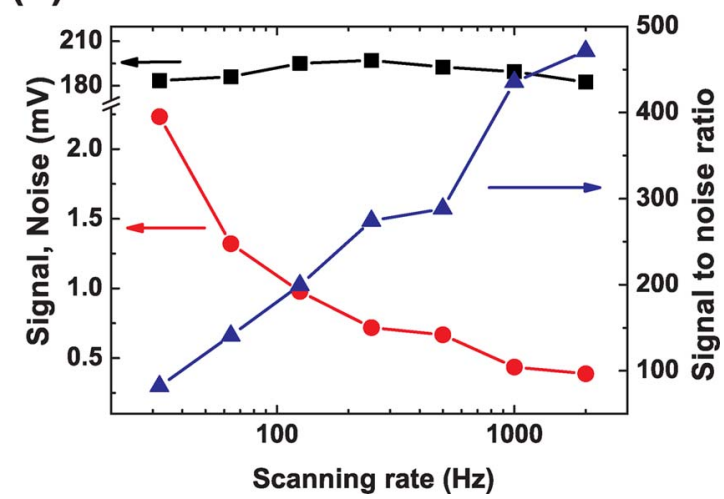

Fig. 3. (Color online) Signal (squares, left vertical scale), noise (circles, left scale), and SNR (triangles, right vertical scale) depending on (a) off-axis alignment parameter at a scanning rate of $1 \mathrm{kHz}$, and on (b) scanning rate at a fixed off-axis diameter of $35 \mathrm{~mm}$. 
(a)
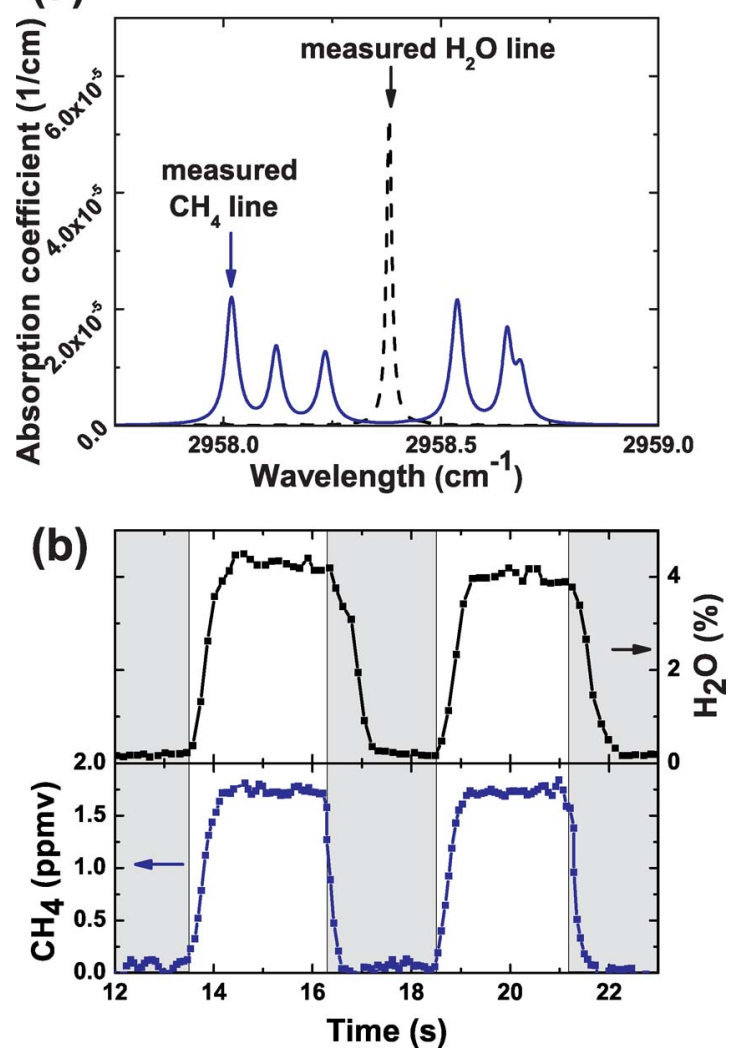

Fig. 4. (Color online) (a) Simulated spectra for $1 \mathrm{~cm}$ path length and a pressure of 250 mbar of 1.7 ppmv of methane (solid curve) and $2 \%$ of water (dashed curve) based on HITRAN 2008 database. (b) Dynamics of water (upper graph, right scale) and methane (lower graph, left scale) concentration measured in exhaled human breath, in real time. White sections correspond to exhalation, gray to inhalation.

The setup was used to measure methane $\left(\mathrm{CH}_{4}\right)$ and water concentrations in exhaled human breath, in real time. Even if the person does not have methanogenic bacteria in his or her intestines that is emitting elevated $\mathrm{CH}_{4}$ levels in the exhaled breath, the exhaled methane concentration will be equal to the inhaled atmospheric methane concentration, which has an average value of 1.7 parts per million by volume (ppmv, $1 \times 10^{6}$ ) [15]. The measurements were performed at the top of the methane line at $2958 \mathrm{~cm}^{-1}$ and the water line at $2958.4 \mathrm{~cm}^{-1}$ at a pressure of $250 \mathrm{mbar}$. The simulation spectra for the $1.7 \mathrm{ppmv}$ of methane and $2 \%$ of water based on the HITRAN 2008 database [16] are shown on the Fig. 4(a). For this experiment, a high-capacity vacuum pump (15 $\mathrm{l} / \mathrm{min})$ was used to keep up with the human exhalation speed. For exhalation, a two-way nonrebreathing T-shape valve (Hans Rudolph Inc.) [17] was used. During inhalation (via the nose), the cell was flushed with nitrogen [Fig. 4(b)].
During exhalation, the nitrogen flow was blocked and exhaled air went into the cell. Figure $4(\mathrm{~b})$ represents the methane and water concentration dynamics during breathing. It clearly shows a subsecond $(0.1 \mathrm{~s})$ time resolution for the measurements.

In summary, the results clearly show that an OPO, pumped by a fiber-amplified diode laser, in combination with OA-ICOS is a powerful tool for the rapid and sensitive detection of trace gases. This approach can be easily extended to multicomponent gas detection and real applications in life sciences. Here, we demonstrate on-line breath sampling that requires detection capability at the ppbv level for multiple gas compounds at a subsecond time resolution.

\section{References}

1. A. Kessler and I. T. Baldwin, Science 291, 2141 (2001).

2. S. M. Cristescu, S. T. Persijn, S. te Lintel Hekkert, and F. J. M. Harren, Appl. Phys. B 92, 343 (2008).

3. S. K. Hetz and T. J. Bradley, Nature 433, 516 (2005).

4. T. H. Rysby, in Disease Markers in Exhaled Breath: Basic Mechanisms and Clinical Applications, N. Marczin and M. H. Yacoub, eds., NATO Advanced Science Institutes Series (IOS, 2002), pp. 113-122.

5. I. D. Lindsay, B. Adhimoolam, P. Gross, M. E. Klein, and K J. Boller, Opt. Express 13, 1234 (2005).

6. A. K. Y. Ngai, S. T. Persijn, I. D. Lindsay, A. A. Kosterev, P. Gross, C. J. Lee, S. M. Cristescu, F. K. Tittel, K. J. Boller, and F. J. M. Harren, Appl. Phys. B 89, 123 (2007).

7. J. A. de Gouw, S. te Lintel Hekkert, J. Mellqvist, C. Warneke, E. L. Atlas, F. C. Fehsenfeld, A. Fried, G. J. Frost, F. J. M. Harren, J. S. Holloway, B. Lefer, R. Lueb, J. F. Meagher, D. D. Parrish, M. Patel, L. Pope, D. Richter, C. Rivera, T. B. Ryerson, J. Samuelsson, J. Walega, R. A. Washenfelder, P. Weibring, and X. Zhu, Environ. Sci. Technol. 43, 2437 (2009).

8. B. W. M. Moeskops, S. M. Cristescu, and F. J. M. Harren, Opt. Lett. 31, 823 (2006).

9. A. O'Keefe, J. J. Scherer, and J. B. Paul, Chem. Phys. Lett. 307, 343 (1999).

10. E. J. Moyer, D. S. Sayres, G. S. Engel, J. M. St. Clair, F. N. Keutsch, N. T. Allen, J. H. Kroll, and J. G. Anderson, Appl. Phys. B 92, 467 (2008).

11. J. Morville, S. Kassi, M. Chenevier, and D. Romanini, Appl. Phys. B 80, 1027 (2005).

12. G. S. Engel, W. S. Drisdell, F. N. Keutsch, E. J. Moyer, and J. G. Anderson, Appl. Opt. 45, 9221 (2006).

13. D. S. Baer, J. B. Paul, M. Gupta, and A. O'Keefe, Appl. Phys. B 75, 261 (2002).

14. J. B. Paul, L. Lapson, and J. G. Anderson, Appl. Opt. 40, 4904 (2001).

15. T. T. Groot, P. M. van Bodegom, F. J. M. Harren, and H. A. J. Meijer, Biogeochem. 64, 355 (2003).

16. HITRAN2008 database http://www.cfa.harvard.edu/HITRAN.

17. F. J. M. Harren, R. Berkelmans, K. Kuiper, S. te LintelHekkert, P. Scheepers, R. Dekhuijzen, P. Hollander, and D. H. Parker, Appl. Phys. Lett. 74, 1761 (1999). 